Dr. Williamson said, that those who were there present ought not to separate without some expression of the pleasure that they had felt on listening to so learned, vast, and eloquent a discourse, treating as it did of a most difficult and important problem. There was scarcely anything of greater moment in the scientific education of youth than the rightly setting before them those wonderful transformations of matter which it is the province of chemistry to explain. These great and growing truths, for, as the lecturer had said, they were growing truths, should be set before youth in such a manner as to form a coherent whole. He hoped to study this masterly discourse with profit and delight, and would now propose a vote of thanks to his illustrious colleague for the honour which he had done them in delivering to them the Faraday lecture.

Prof. Tyndall said he had heard the discourse with deep interest, for it showed that the lecturer knew the importance of a teacher's vocation, and that his province was not merely to communicate knowledge, but to do it in such a manner as to arouse an interest in and love of the subject in the pupil by presenting it in its proper relations. $\mathrm{He}$ would have welcomed the lecturer to that Institution, even had he come to tear in pieces the notions which he cherished regarding atoms and molecules; how pleasant it was then to find such a broad agreement between their views. The chemist cannot halt at equivalent proportions - he must ask himself whence they arise, and the inevitable answer is some form of the atomic theory. This theory, however, cannotbe confined to chemical phenomena. The motions of those atoms and molecules underlie all our explanations of the physical cause of light and heat, and it is already taking up the field of magnetism and electricity. Consider, for example, the heat of gases, both as regards the motion of translation of the molecules which produce temperature, and the motions of rotation and vibration of their constituent atoms, which, though they do not express themselves as temperature, constitute a portion of the heat. Clausius has shown that even in the simplest gases nearly two-fifths of the whole heat is due to these internal motions; while in gases of complex molecular constitution which condense on com bining, the ratio of the total heat to the heat of temperature is still greater. The experiments of Regnauli, which show that the specific heat of a perfect gas at a constant volume is constant, proves, as Clausius has shown, that the one kind of motion is proportional to the others.

The lecturer had also referred to atoms of the same kind combining together, so that, free oxygen and free hydrogen being considered as composed of molecules each containing a pair of atoms, has certainly simplified the results. But it must not be forgotten that this combination of like atoms is widely different from that of unlike atoms. The union of oxygen with oxygen or nitrogen with nitrogen produces no such effects upon the luminiferous ether as the union of oxygen with nitrogen. With the same quantity of matter the amount of vis viva sent forth as radiant heat may be augmented a thousandfold, perhaps a millionfold, by the act of diverse combination. This act seems to carry with it a condensation of the ether to a dense atmosphere around the atoms. Had a cannon the power of gathering round itself a dense atomsphere, it would send forth a greater amount of zis viva as sound. $A$ gun fired at Chamouni may be heard upon Mont Blanc while the same gun fired on Mont Blanc may not be heard at Chamouni, because the air on which the concussion takes place is denser in the one case than in the other. In the same way the diverse atoms vibrating in the denser atmosphere formed on combination show their vast superiority as radiators over like atoms which, except in such special cases as ozone, \&c., are incompetent to produce a similar condensation. The speaker then asked them to echo the resolution so well put to the meeting by Prof. Williamson.

\section{THE OBSERVATORY ON MOUNT VESUVIUS}

W

HILE the scientific world and his own countrymen are rivals in doing honour to Prof. Palmieri for his zeal in remaining at his post in spite of all danger, it may be interesting to examine in some detail the work done at the Observatory of Mount Vesuvius. We know wonderfully little about the origin and mutual dependence of volcanic phenomena. This is due to a want of accurate observations. For the complete investigation we require first to know at what dates earthquakes and eruptions occur at different parts of the earth. Next we must have observations of the direction and exact hour at which a wave of disturbance passes different places whose positions are known. This gives us the velocity of the wave, and helps to determine the position, under the earth's surface, of the centre of disturbance; or, if a wave be propagated over the sea, we obtain a means of estimating the average depth of the intervening ocean; for the velocity of a wave increases with the depth of the sea. This method gives one of the best determinations we possess of the depth of the Pacific Ocean. But beyond this we must have observations made systematically at some place subject to earthquakes and volcanic eruptions. No place in Europe is more suitable for this than the neighbourhood of Mount Vesuvius ; and it was for such observations that an Observatory was established there.

Everyone knows that Mount Vesuvius consists of a great cone of lava and ashes, at the top of which is the great crater. On the northern side, separated from it by the deep valley called the Atrio del Cavallo, rises the precipitous and semicircular Monte Somma. This once formed the crater of the volcano, and the present cone seems to have been formed inside that great crater at the time when Pompeii was overwhelmed. On a spur of rock, a mile or two in length, running down from the Atrio del Cavallo, the Observatory is placed. It is close to the well-known "Hermitage," or half-way house, in the ascent of the mountain. Being raised on this ridge above the surrounding country, it is comparatively safe from the molten lava that flows at times on either side of it.

The building itself is handsome; in fact it is to be regretted that so much money should have been devoted to the masonry instead of to additional instruments. On the ground floor are the inhabited rooms, all scantily furnished; but the pursuers of science cannot always expect bodily comfort. On the first floor we find the Museum, with a fire collection of minerals found on the mountain. Perhaps it may be as well here to correct a common mistake as to the nature of the yellow substance found about the craters, whose brilliant colours remind one so much of the Solfatara. This substance is not sulphur, but copper. The most interesting objects in the Museum are the "fumerolles," or smoke-holes. Occasionally at the end of an eruption you may see at the bottom of the crater a small cone of lava, with a hole in its top, through which the steam pours with a hissing noise like a wave breaking on a pebbly beach, or like a blast furnace, or as Pliny has it, like the grinding of a saw ; the intensity of the sound varying with your position. These small cones are the fumerolles; they are a foot or two high; and Palmieri has actually had several of these natural chimneys cut off and transported to the Museum.

We now pass on to the Observing Room. There are solid piers carried up from the ground to support the in. struments. First comes the elegant seismograph for the automatic registration of earthquake shocks. The object of the instrument is twofold : first to measure the direction and intensity of a shock; and, second, to write down a history of the earthquake. The shock may be either vertical or horizontal, or partly vertical and partly horizon, tal. For the vertical shocks a fine metallic point is suspended by a coil of wire over a cup of mercury. The coil of wire acts as a spring, and the slightest upward motion of the 
e arth is sufficient to cause the point to dip into the cup of mercury. This completes a galvanic circuit, which stops a clock at the exact half second at which the shock occurred, and rings a bell to call the observer, and also does other work which we shall speak of again. There are three or four helices of wire of different strengths, which support small magnets above a cup of iron filings. When a vertical shock occurs some of these magnets dip into the iron filings. To one of these a light index is attached for measuring the intensity of the shock.

For horizontal shocks there are four glass tubes. Each of them is bent twice at right angles, so as to form a $U$ tube. One arm of this tube has more than double the diameter of the other, and is shorter. The four tubes point inthe directions of the four cardinal points. Each tube has a certain quantity of mercury poured into it, and on the surface of the mercury, within the narrow arm of the tube, there rests a small weight attached to a silk fibre, which passes over a delicate ivory pulley, and has a counterpoise attached at the other end. Each pulley has an index and circular scale to mark the angle turned through. The extremity of a wire is fixed at a small distance above the surface of the mercury in each tube. If then a horizontal shock occur, the mercury rises in the corresponding tube ; but it rises higher in that one which has its long arm to the north. The pulley is turned through a certain angle, which is measured by the index, and at the same time the mercury in rising comes in contact with the fixed wire, and so completes a galvanic circuit which rings a bell, and stops the clock at the exact half second when the shock occurred. If the shock comes from some intermediate point two of the indices will be moved, and the direction and intensity can be measured by observing both of them. We have seen up to this point that the instrument will measure the direction and intensity of a shock, will mark the time at which the shock occurred, and will ring a bell to attract the attention of the observer on duty, who may register succeeding shocks, or, if the earthquake has ceased, may reset the apparatus. But this is not all. The galvanic circuit, which is completed at the moment a shock occurs, releases at the same instant the pendulum of a second clock, which has been held out of the vertical by means of a detent. This clock allows a roll of paper to be unwound off a drum, as in any registering telegraph, at the rate of three metres an hour. A pencil rests nearly in contact with the strip of paper. It is connected with one arm of a lever, the other arm of which is slightly distant from an electro-magnet. As often as the current passes this end of the lever is attracted to the magnet, and the pencil in consequence is made to press on the paper, to be released only when the current ceases. By this means then a continuous history of the earth's trembling is registered, a pencil mark corresponding to a time of trembling, and a blank space to a period of cessation.

This instrument is extremely delicate, and registers motions of the earth which are too slight to be perceptible to the human frame. When we examined it some one happened accidentally to touch the casing of the instrument. The alarm was immediately given by the bell, and the two clocks were respectively checked and put in motion by the galvanic current.

The accompanying figure (borrowed from the $E n$ gineer, for the use of which we are indebted to the courtesy of the editor of that journal) may help to make the above description more intelligible. In Fig. I, the clock $\mathrm{A}$ is shown with the pendulum arrested, as after a shock has occurred. The pendulum of the clock $B$ is in a position ready to be set free when a shock occurs. At the same time the strip of paper $k k k$ will be rolled on to the cylinder $i$, and at each trembling of the earth the electro-magnet $D$ will cause a pencil to make a mark on the paper at the point $m$. $\mathrm{P}$ and $\mathrm{R}$ are two gillars, between which are shown the $U$ tubes con- taining the mercury, the pulleys and indices are shown above. These pillars and tubes are also shown in plan. Metallic bars are seen connected with $R$, and passing over the short arms of the tubes. From these hang the wires that dip into the mercury. From the pillar $P$, metallic bars are also shown passing over the long arms of the tubes ; to these are attached the wires that are almost in contact with the mercury, and which complete the circuit when a shock occurs. The metallic spring E, supported by the pillar $T$, above the cup of mercury $f$, is the apparatus for making a current during a vertical shock; $h h h$ are the springs with magnets attached, which dip into iron filings. The index for vertical shocks is shown more clearly in Fig. 2 .

For more violent shocks the heavy bob of a freely suspended pendulum is placed in the centre of a horizontal ring in which eight tubes are placed lightly, all pointing to the centre. From whatever direction a horizontal shock comes it will drive out one of these tubes. The tube driven out will show the direction of the shock, and the distance to which it is driven will show the intensity. This is also shown in plan (Fig. 3). The hole for the tube is also represented. There is also shown in section a cup of mercury, placed at the foot of the pillar $G$ (Fig. I), which has eight holes in its circumference just above the surface of the mercury. When a shock occurs mercury is driven out into the hole corresponding to the direction of the shock. The quantity of mercury determines the intensity. The battery is shown in Fig. 4 and needs no explanation.

In the same room there is apparatus for detecting and measuring atmospheric electricity. A gold leaf electroscope and a bifilar electrometer are observed regularly. These are successively put in connection with the conductor. This consists of a disc of metal above the roof of the house connected with an insulated metallic rod, supported vertically, and capable of being rapidly raised by means of a cord passing over a pulley. When not in use this rod is in connection with the ground. In making an observation the rod with the disc attached is quickly raised, thereby disconnecting it from the ground. The electricity of the atmosphere at the point where the disc is fixed affects the electroscope and electrometer. Prof. Palmieri prefers the conductor above-described to a conducting point or a flame, because he considers that these do not give comparable results, an objection which is not supported by all observers. He considers the same to be true of the method of dropping water.

After having made careful observations on atmospheric electricity for about a quarter of a century in a country where meteorological changes are more regular and less capricious than in our own island, there is no one whose deductions are more deserving of our attention; the more so as he considers that he has combined his researches into a definite law. His first fact is this:-If within a distance of about fifiy miles there is no shower of rain, hail, or snow, the electricity is always positive. The single exception is during the projection of ashes from the crater of Vesuvius. During a shower he finds the following law universally to hold good:-At the place of the shower there is a strong development of positive electricity; round this there is a zone of negative, and beyond this again positive. The nature of the electricity observed depends upon the position of the observer with respect to the shower, and the phenomena will change according to the direction in which the shower is moving. Sometimes negative electricity may be observed during a shower; but this is always due to a more powerful shower farther off. These conclusions have been supported by means of telegraphic communication with neighbouring districts. It appears, then, that except when the moisture of the air is being condensed, there is no unusual development of electricity. These results are in accordance with the experiments of Palmieri and others, which show that 


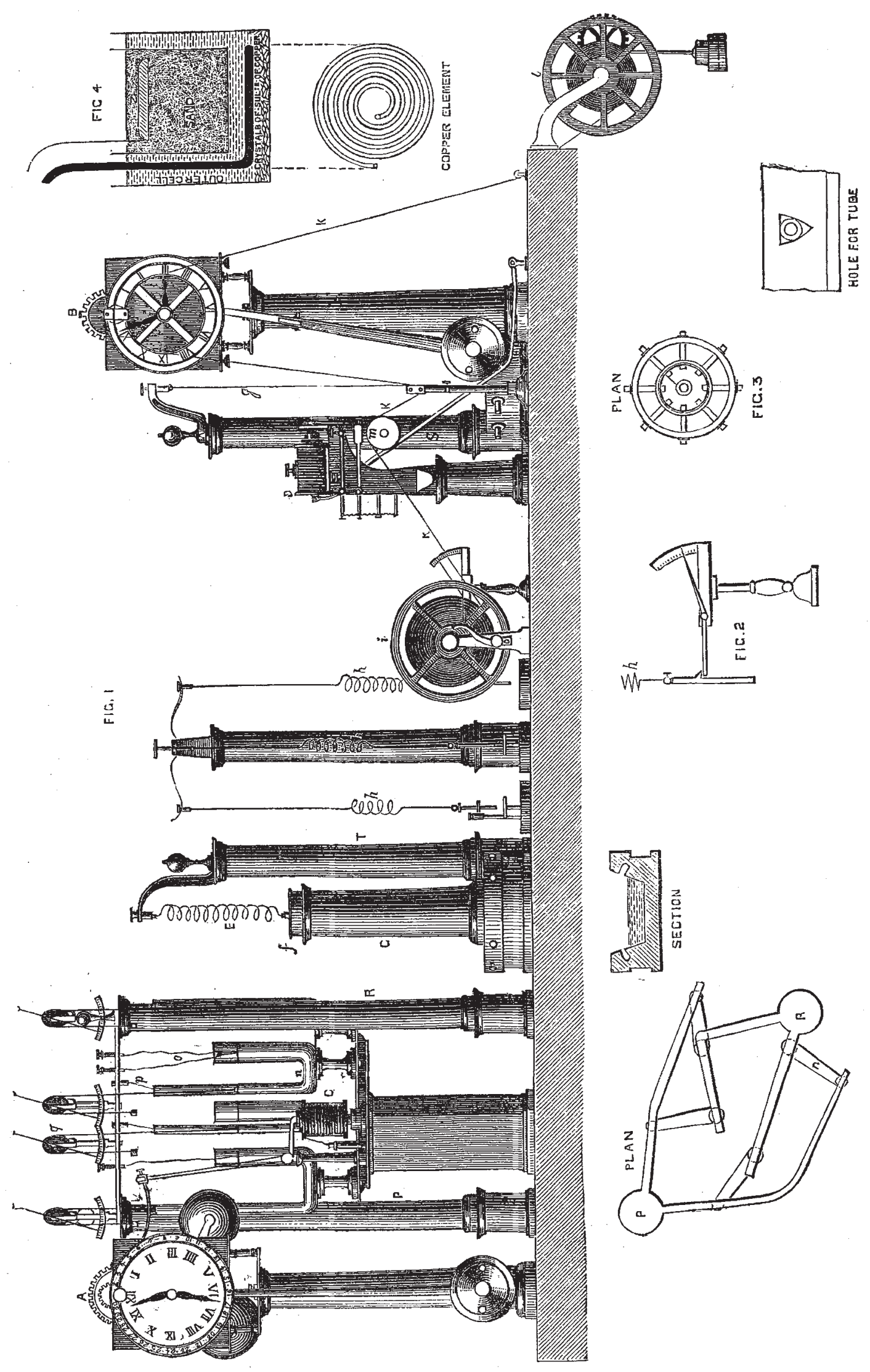


aqueous vapour in condensing developes positive electricity. No unusual development of electricity has ever been detected by him in a cloud when no rain is falling.

The above results, though falling short of what has to be done to complete the theory, are yet definite, and hence valuable, the more so if supported by other observers placed in equally favourable situations. But of the variations in intensity of positive or negative electricity nothing has been said.

Besides the fixed instruments at the Observatory others are used on the mountain. Gases are collected from cracks in the earth's crust, tubes being let down into them, and the gas sucked up by a kind of bellows to be examined at leisure. A portable spectroscope is also used during eruptions, and there is a larger one by Hoffman in the Observatory. From this Observatory we have received valuable information, and it is much to be regretted that equally efficient observatories have not been established in different parts of the world. Many portable and cheap instruments have been invented, most of which are described by Mr. Mallet in the "Admiralty Manual of Scientific Inquiry ;" but there ought to be three or four as delicate as that on Mount Vesuvius. It is a pity that no observatory has ever replaced the ancient one of Empedocles near the summit of Etna, or even at Nicolosi, where the valuable services of Dr. Gemellaro might have been obtained. This would have been the more interesting, as Palmieri can detect shocks caused by that volcano, though the distance is enormous. With a third observatory, say in the Philippine Islands, we could not fail to increase our knowledge enormously.

From long practice Palmieri is able to predict eruptions. We remember well when we were enjoying his hospitality at the beginning of last year how he said, "This is a small eruption, but there is going to be a great one ; I do not say it will be soon, it may be a year, but it will come." In almost exactly a year the great eruption did come.

GEORGE FORPES

\section{ON THE DISINTEGRATION OF COMETS}

$r \mathrm{HE}$ main design of the following paper is to present 1 at one view the historical evidence of the gradual disintegration of periodic comets. A few preliminary remarks, however, in regard to the received theory of comets and meteors, may not be destitute of interest.

The fact that in several instances meteoric streams move in orbits identical with those of certain comets was first fully established by the researches of Signor Schiaparelli. The theory, however, of an intimate relationship between comets and meteors was proposed and advocated by the writer several years previous to the publication of Schiaparelli's memoirs. In an article written in July $\mathbf{1 8 6 1}$, and published in the "Danville Quarterly Review" for December of that year, it was maintained-

I. That meteors and meteoric rings "are the débris of ancient, but now disintegrated comets whose matter has become distributed around their orbits."

2. That the separation of Biela's comet as it approached the sun in December 1845 was but one in a series of similar processes which would probably continue until the individual fragments would become invisible.

3. That certain luminous meteors have entered the solar system from the interstellar spaces. $t$

4. That the orbits of some meteors and periodic comets have been transformed into ellipses by planetary perturbation. And-

5. That numerous facts-some observed in ancient and some in modern times-have been decidedly indicative of cometary disintegration.

What was thus proposed as theory has been since confirmed as undoubted facts. When the hypothesis was

* The name of cometoids was accordingly proposed for luminous meteors. t Others, it was supposed, might have originated within the systemview which the writer has not wholly abandoned. originally advanced, the data required for its mathematical demonstration were entirely wanting. The evidence, however, by which it was sustained was sufficient to give it a high degree of probability.

The existence of a divellent force by which comets near their perihelia have been separated into parts, is clearly shown by the facts enumerated in the following lines. Whether this force, as suggested by Schiaparelli, is simply the unequal attraction of the sun on different parts of the nebulous mass, or whether, in accordance with the views of other astronomers, it is to be regarded as a cosmical force of repulsion, is a question left for future discussion.

I. Seneca informs us that Ephoras, a Greek writer of the fourth century B.C., had recorded the singular fact of a comet's separation into two distinct parts. * This statement was deemed incredible by the Roman philosopher, inasmuch as the occurrence was then without a parallel. More recent observations of similar phenomena leave no room to question the historian's veracity.

2. The head of the great comet of 389 A.D., according to the writers of that period, was "composed of several small stars" (Hind's "Comets," p. ro3).

3. On June 27, A.D. 416, two comets appeared in the constellation Hercules, and pursued nearly the same apparent path. Probably at a former epoch the pair had constituted a single comet. $\dagger$

4. On Aug. 4, 8I3, "a comet was seen which resembled two moons joined together." They subsequently separated, the fragments assuming different forms.+

5. The Chinese annals record the appearance of three comets-one large and two smaller ones-at the same time in the year 896 of our era. "They travelled together for three days. The little ones disappeared first, and then the large one." \$ The bodies were probably fragments of a large comet which, on approaching the sun, had been separated into parts a short time previous to the date of their discovery.

6. The third comet of I6I8.-The great comet of I6I8 exhibited decided symptoms of disintegration. When first observed (on November 30), its appearance was that of a lucid and nearly spherical mass. On the eighth day the process of division was distinctly noticed, and on the 2oth of December it resembled a cluster of small stars.

7. The comet of $166 \mathrm{r}$. - The elements of the comets of I532 and I66I have a remarkable resemblance, and previous to the year 1790 astronomers regarded the bodies as identical. The similarity of the elements is seen at a glance in the following table :-

\begin{tabular}{|c|c|c|}
\hline Longitude of Perihelio & $\begin{array}{l}\text { Comet of } 15,32 . \\
\text { - } \quad \text { I I I }{ }^{\circ} 48^{\prime}\end{array}$ & $\begin{array}{c}\text { Comet of } 166 x \\
\text { I I } 5^{\circ} 16^{\prime}\end{array}$ \\
\hline Longitude of Asc. Noc & . 8723 & 8 I 54 \\
\hline Inclination . . & 3236 & 33 \\
\hline Perihelion Distance & 0.5192 & 0.4427 \\
\hline Motion . . . . & - Direct & Direct \\
\hline
\end{tabular}

The elements of the former are by Olbers; those of the latter by Mechain. The return of the comet about 1790 , though generally expected, was looked for in vain. As a possible explanation of this fact it is interesting to recur to an almost forgotten statement of Hevelius. This astronomer observed in the comet of 1661 an apparent breaking up of the body into separate fragments. If The case may be analogous to that of Briela's comet.

8. The identity of the comets of 1866 and 1366 , first suggested by Prof. H. A. Newton, is now unquestioned. The existence, then, of a meteoric swarm, moving in the same track, is not the only evidence of the original comet's partial dissolution. The comet of 1866 was invisible to the naked eye; that of 1366 , seen under nearly similar * "Quæst., Nat.," lib. vii., cap. xvi.

t Chambers's "Descr. Astr.,"p. 374

Ibid. p. 383 .

Hevelius, "Cometographia," p. 34x. See also Grant's "History of Physical Astronomy," p. 302. 